\title{
Voila, a New Drosophila Courtship Variant that Affects the Nervous System: Behavioral, Neural, and Genetic Characterization
}

\author{
Maria Balakireva, ${ }^{1}$ Reinhard F. Stocker, ${ }^{2}$ Nanaë Gendre, ${ }^{2}$ and Jean-François Ferveur ${ }^{1}$ \\ 1 Unité de Recherche Associée Centre National de la Recherche Scientifique 1491, Université Paris-Sud, 91405 Orsay- \\ Cedex, France, and 2Institute of Zoology, University of Fribourg, CH-1700 Fribourg, Switzerland
}

\begin{abstract}
In Drosophila melanogaster, a specific PGAL4 transposon induces the Voila ${ }^{1}$ genetic variant and produces multiple phenotypes. Homozygous Voila $^{1 / 1}$ flies rarely reach adulthood, whereas heterozygous Voila ${ }^{1 /+}$ adult males show strong bisexual behavior. Males with a single copy of Voila ${ }^{1}$ driving the feminizing transgene UAS-transformer show very reduced sexual activity but no overall effect on their behavior. Voila ${ }^{1}$ is specifically expressed in the nervous system. In the CNS, it is expressed mainly in the mushroom bodies and, to a lesser
\end{abstract}

extent, in the antennal lobes. In the peripheral nervous system, GAL4 expression is almost entirely restricted to the gustatory sensilla. Using chromosomal deficiencies, the behavioral alteration was genetically mapped to the same location as the PGAL4 element $\left(86 \mathrm{E}_{1-2}\right)$. The multiple behavioral effects of the Voila genetic variant are discussed in light of its expression in the nervous system and its genetic basis.

Key words: Drosophila; courtship behavior; PGAL4; gustation; mushroom bodies; transformer
Understanding the function of genes and the neural circuitry that underlies complex behaviors has been the focus of increasing scientific interest over the past decade. In Drosophila melanogaster, studies of courtship offer an explanation of the ways genes influence the execution of complex behaviors (Greenspan, 1995).

Sexually mature $D$. melanogaster males perform elaborate courtship behaviors in response to conspecific females (Sturtevant, 1915). Mature males generally court female and immature flies, but in some circumstances they also vigorously court conspecific mature males (Ferveur et al., 1995, 1997; Zhang and Odenwald, 1995; Yin Hing and Carlson, 1996). During a courtship bout, the male orients toward his partner and taps her (or his) abdomen with his forelegs. Next, he will vibrate one wing, thereby producing a species-specific song. Then, he may lick the genitals of his partner, curl his abdomen, and attempt copulation. These stereotypic precopulatory behaviors are sex-specific and genetically determined (Hall, 1994).

In $D$. melanogaster, very few genes are known that affect only courtship. In the genetic hierarchy of sex differentiation, Sexlethal (Sxl) and transformer (tra) control sexual behavior as part of their larger influence on somatic sex determination (Sturtevant, 1945; Tompkins and McRobert, 1989, 1995). Downstream of tra, doublesex and fruitless ( $\mathrm{fru}$ ) control two independent pathways of sexual differentiation (Taylor et al., 1994; Villela and Hall, 1996).

\footnotetext{
Received Sept. 15, 1997; revised March 10, 1998; accepted March 12, 1998.

This work was partly supported by the Human Frontier Science Program Grant RG 93/94 to M.B., R.F.S., and J-F.F., by the French Lilly Foundation Grant 97-98 to M.B., and by the Swiss National Science Foundation Grant 31-42053.94 to R.S. We are grateful to Dr. Klemens Störtkuhl (Ruhr-Universitaet, Bochum) for his help and advice in the PGAL4 screen that led to the isolation of line DB345, and to Dr. Françoise Lemeunier (Centre National de la Recherche Scientifique, Gif-surYvette) for her precious help in the in situ chromosomal localization. pBM292 plasmid that contains the GAL4 sequence was kindly provided by J. Urban (Mainz University), and the UAS-tau strain was provided by T. Raabe (Würzburg University). Matthew Cobb and two anonymous reviewers are thanked for their comments on this manuscript.

Correspondence should be addressed to Jean-François Ferveur, Unité de Recherche Associée Centre National de la Recherche Scientifique 1491, Bâtiment 446, Université Paris-Sud, 91405 Orsay-Cedex, France.

Copyright (ㄷ) 1998 Society for Neuroscience $0270-6474 / 98 / 184335-09 \$ 05.00 / 0$
}

fru acts specifically on the CNS (Ryner et al., 1996), and various fru mutant alleles diversely affect male courtship behavior (Ito et al., 1996; Villella et al., 1997). One of the most conspicuous behavioral phenotypes exhibited by fru males is the courtship chain of male flies in which individuals are simultaneously courters and courtees. Recently, the dissatisfaction ( $d s f)$ gene has been shown to specifically affect-downstream of tra but independently of $d s x$-sex-specific courtship behaviors and neural differentiation in flies of both sexes (Finley et al., 1997).

A pioneering technique that produced mosaic flies with $\mathrm{XX}$ and XO tissues was initially used to map the neural structures that control male courtship (Hall, 1977, 1979; von Schilcher and Hall, 1979) and female receptivity to male courtship (Tompkins and Hall, 1983). It is now possible to feminize small subsets of the male brain by ectopically expressing the female form of the sex-determination gene tra (UAS-tra), under control of PGAL4 enhancer-trap lines. The partial feminization of the antennal lobes (ALs) or the mushroom bodies (MBs) or both causes mosaic males to engage in bisexual courtship (Ferveur et al., 1995; O'Dell et al., 1995). These results suggest that MBs and ALs differ functionally between the sexes. The MBs, which are neurally connected with the ALs, contain a sexually dimorphic number of neurons (Technau, 1984) and express male-specific genes such as roX1, independently of tra activity (Amrein and Axel, 1997; Meller et al., 1997). The prothoracic legs, the maxillary palps, and the antenna also show a different number of chemosensilla in the two sexes (Nayak and Singh, 1983; Singh and Nayak, 1985; Venard et al., 1989; Stocker, 1994). In particular, primary afferents from the leg sensilla project with a sexually dimorphic pattern into the thoracic ganglia (Possidente and Murphey, 1989).

Mate recognition relies on sexually dimorphic signals and especially on cuticular hydrocarbons that act as sex pheromones (Antony and Jallon, 1982; Jallon, 1984). Drosophila males are able to simultaneously perceive female and male predominant pheromones: female pheromones stimulate male sexual excitation, whereas male-specific molecules tend to inhibit male excitation 
(Ferveur and Sureau, 1996). Pheromones are mainly detected by contact during tapping and licking, pinpointing the crucial role of gustatory organs borne by the prothoracic legs and the proboscis (Ferveur, 1997). A recent experiment shows that the sexual orientation of mosaic XY flies is independent of their pheromonal feminization (Ferveur et al., 1997).

Here we describe the behavioral, neuronal, and genetic characteristics of the new genetic variant Voila, which may play a crucial role in pheromonal perception and male courtship behavior. Voila is the first reported case of a complete block of courtship correlated with manipulation of a restricted part of the nervous system.

\section{MATERIALS AND METHODS}

Fly stocks and genetics. Fly stocks were maintained at $25^{\circ} \mathrm{C}$ in a $12 \mathrm{hr}$ dark/light cycle. Crosses were performed using standard techniques. A description of the chromosomes and mutations used in this study can be found in Lindsley and Zimm (1992).

The PGAL4 DB345 insertion line (carrying Voila ${ }^{1}$ ) was isolated in an enhancer-trap screen that focused on the fly chemosensory system (DB345 was kindly provided by Damina Balmer, Fribourg). Because the Voila $^{1}$ insert is recessive lethal, the Voila ${ }^{1}$ chromosome was maintained balanced over the chromosome TM3, Sb Ser. To produce (shi) Voila $1 /+$, a strain built with the genetic background of the shibire $e^{\text {ts }}$ strain (shi), Voila $^{1} /$ TM3 males were crossed with shi females, and subsequent heterozygous Voila ${ }^{1} /+$ male progeny were backcrossed to shi females for five successive generations. Neither males nor females from the (shi) Voi$l a^{1} /+$ strain carry the $\mathrm{X}$ chromosome associated with the thermosensitive $s h i^{\text {ts }}$ mutation. We also backcrossed Voila ${ }^{1}$ into other genetic backgrounds: flies from these strains yielded the same behavioral tendency, although it was weaker than in (shi) Voila $1 /+$.

Genetic mapping of the behavioral defect caused by the Voila ${ }^{1}$ insert was performed with deficiencies covering the chromosomal region between $86 \mathrm{C}$ and $87 \mathrm{C}$ (for information about their breakpoints and origin, see Reuter et al., 1987).

Females from the shi strain and Canton-S (CS) males were chosen as control objects because of their clear behavioral phenotypes (Ferveur and Sureau, 1996; Ferveur et al., 1997).

Behavioral tests. Behavioral tests were performed using a protocol modified from Ferveur and Sureau (1996). Briefly, all behavioral assays were performed on 4-d-old males (kept alone after eclosion) that were individually aspirated into an observation chamber $(2.8 \mathrm{~cm}$ diameter, 0.5 $\mathrm{cm}$ height). After $10 \mathrm{~min}$, a 4-d-old virgin shi female or a CS male, used as the object fly, was introduced. The courtship index value $(C I)$ is the percentage of time that the subject male spends courting during a $10 \mathrm{~min}$ observation period. We noted the occurrence of different stereotypical male behaviors: tapping, wing vibration, licking the genitalia, and attempted copulation. We also noted the frequency with which subject males attempted copulation with an object fly. Object flies were generally decapitated (except when otherwise indicated).

Decapitation prevents reciprocal courtship and standardizes the duration of courtship, because no copulation occurs with decapitated females (Ferveur et al., 1995). Courtship indices were arc sin-transformed before being compared statistically. The data were not always normally distributed, probably because there was a high proportion of males that did not show any homosexual courtship $(\mathrm{CI}=0)$. The effect of the genotype of both flies (courter and courtee) and their interaction was measured with a two-factor ANOVA. Using the same procedure, the effect of decapitation was separately tested on male or female object flies. We decided not to mix the three factors in the same analysis because the "decapitation-intact" factor produces very different behavioral consequences in the two sexes: copulation occurs only in intact females.

Locomotor activity was measured in similar environmental conditions. We averaged the total number of lines, drawn under the chamber, crossed by each fly (locomotor activity units = LAU) [modified from Tompkins et al. (1982)]. For each experiment, four single flies were sequentially observed for five periods of $20 \mathrm{sec}$, every $2 \mathrm{~min}$. At least 25 flies per genotype were studied. Locomotor activity values were normally distributed and thus were compared with a Student's $t$ test.

The sexual receptivity of females was measured by latency to copulation when paired with control males. Logarithmically transformed $\left(\log _{\mathrm{e}}\right)$ latencies were statistically compared between experimental and control females (Mann-Whitney $U$ test).
Histochemistry and immunochemistry. The Voila ${ }^{1} / T M 3$ strain was crossed to a UAS-lacZ strain (Brand and Perrimon, 1993), to a UAS-tau strain (Ito et al., 1997), or to a UAS-GFP strain (Brand, 1995). The transactivated $\beta$-galactosidase was visualized either histochemically or immunocytochemically. In the first case, heads, thoraces, and abdomens of the Voila ${ }^{1} U A S$-lacZ heterozygotes were dissected in Millonig's buffer, fixed in $1 \%$ glutaraldehyde (in Millonig's buffer), and stained for $\beta$-galactosidase activity with a solution containing 5-10 $\mathrm{mg} \mathrm{X-Gal} / \mathrm{ml}$ DMSO (Brand and Perrimon, 1993). The CNS, heads, thoraces, wings, and legs were mounted in Faure's solution. For immunocytochemistry, a monoclonal anti- $\beta$-galactosidase antibody (Promega, Madison WI) was applied 1:2000 overnight at $4^{\circ} \mathrm{C}$ to $10 \mu \mathrm{m}$ cryosections and then stained with the Vectastain ABC Kit (Vector Laboratories, Burlingame CA). The tau protein in Voila ${ }^{1}$ UAS-tau heterozygotes was tagged by a monoclonal anti-tau antibody (Sigma, St. Louis MO) (1:2000) and stained in the same way as for $\beta$-galactosidase. GFP patterns were visualized in a Bio-Rad MRC 1024 confocal microscope.

In situ chromosomal mapping. Polytene chromosome squashes from $w$; Voila $^{1} /+$ heterozygotes were prepared using the procedure of Zuker et al. (1985). The position of the PGAL4 insertion was determined using the biotinylated DNA probe of the pBM292 plasmid, which contains the GAL4 sequence. In situ hybridization to polytene chromosomes was performed according to Smith et al. (1990). The DNA of the pBM292 plasmid was biotinylated using a nick-translation system (BRL, Bethesda, MD) and biotin-11-dUTP and detected by the binding of streptavidincoupled alkaline phosphatase (Enzo Biochemicals).

\section{RESULTS}

\section{The bisexual orientation of Voila ${ }^{1}$ segregates with the chromosome 3 carrying the PGAL4 transposon}

The strain DB345 is an enhancer-trap strain that we induced by PGAL4 mutagenesis (Brand and Perrimon, 1993). Males with a single copy of the PGAL4 DB345 transposon show abnormal courtship behavior: they actively court both virgin females and mature males. The locus containing the PGAL4 insertion is associated with altered male sexual orientation and was named Voila (à la voile et à la vapeur, French slang for a bisexual human).

Mutant males of the original strain Voila $1 /+$ and of the balanced strain Voila ${ }^{1} / T M 3$, exhibited a vigorous bisexual courtship toward both intact and decapitated flies (Fig. 1). Voila ${ }^{1} /$ TM3 males were still able to discriminate between intact females and males: their courtship intensity was higher with females $\left(C I_{\mathrm{f}}=83\right)$ than with males $\left(C I_{\mathrm{m}}=35\right)$. Furthermore, Voila ${ }^{1} / T M 3$ males attempted to copulate more often with intact female $(83 \%)$ than with intact male partners $(18 \%)$. When paired with decapitated targets, Voila ${ }^{1} /$ TM males also showed very vigorous bisexual courtship responses $\left(C I_{\mathrm{f}}\right)$ $\left.C I_{\mathrm{m}}=66 / 38\right) ; 73 \%$ and $29 \%$, respectively, of these males attempted to copulate with decapitated female or male flies.

The chromosome 3 carrying the Voila ${ }^{1}$ insert, outcrossed in the background of the control shibire strain [(shi) Voila ${ }^{1} /+$; see Materials and Methods], segregated with a strong male bisexual orientation: (shi) Voila $1 /+$ males exhibited courtship responses that were very similar to those of Voila ${ }^{1} / T M 3$ males. With intact targets, their courtship indices $\left(C I_{\mathrm{f}} / C I_{\mathrm{m}}\right)$ were $93 / 49(81 / 43$, with decapitated flies); $84 \%$ and $21 \%$ of male flies attempted to copulate with intact female and male partners, respectively (95\% and $18 \%$, with decapitated targets). In comparison, control CS males performed highly active heterosexual courtship with females, but very little homosexual courtship $\left(C I_{\mathrm{f}} / C I_{\mathrm{m}}=72 / 3\right.$ with intact flies and $47 / 3$ with decapitated flies).

Statistical analysis reveals that the genotype of the courter and the sex of the courtee, but not their interaction, significantly affect the intensity of courtship (Table 1). This suggests that subject males from the three strains show the same degree of sexual discrimination (ability to discriminate the sex of their partner). Also, decapitation exerts a significant behavioral effect when tested with object females, but not with males. 
A

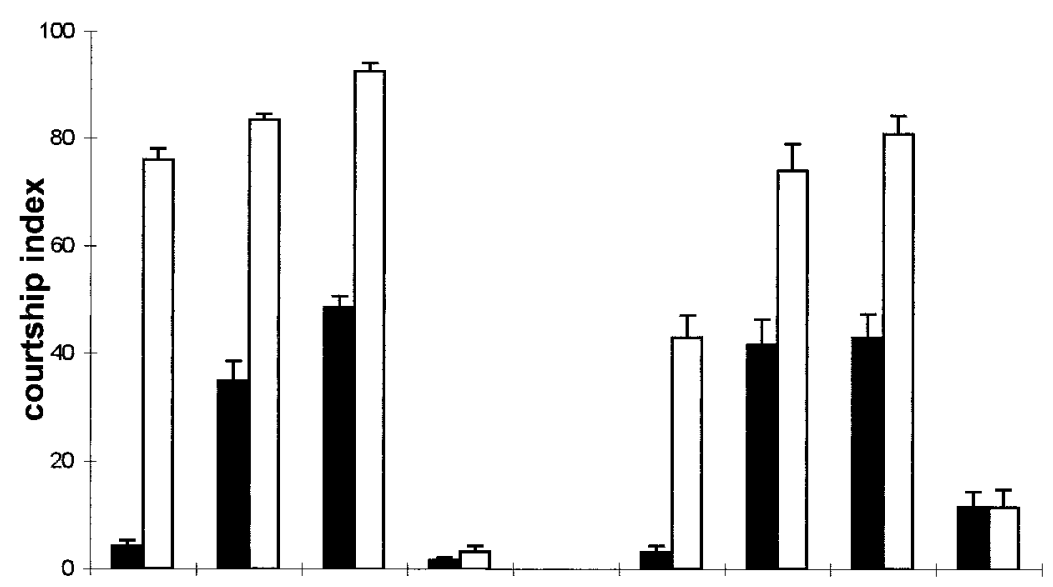

B
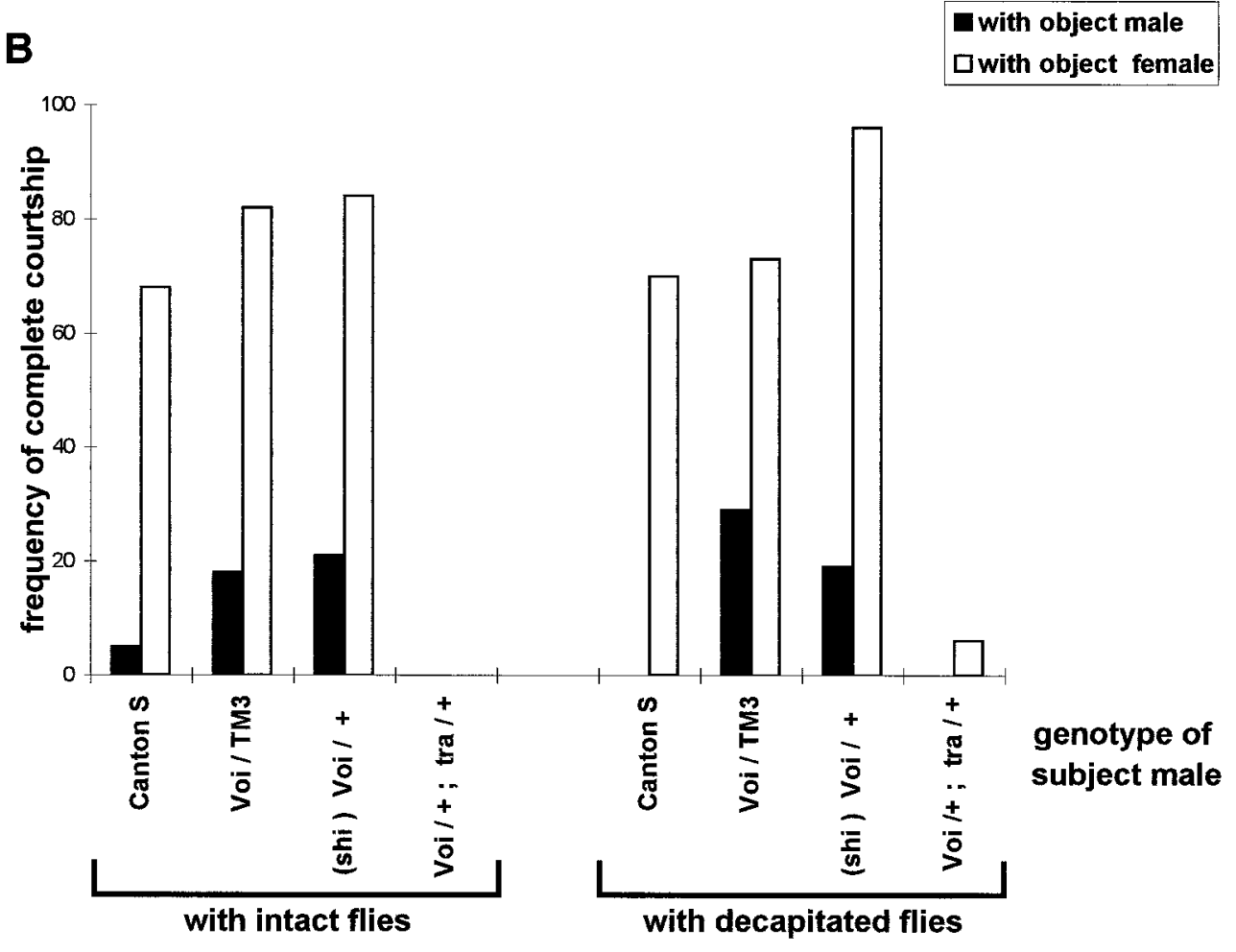

Figure 1. Courtship behavior of Voila ${ }^{1}$, Voila ${ }^{1}$ UAS-tra, and control males. The courtship directed toward intact and decapitated male or female targets is expressed as the courtship index $(A)$ or as the frequency of complete courtship $(B)$. For each genotype, the courtship of 35 and 20 males was measured with male and female targets, respectively. For description of genotypes, see Material and Methods. $A$, Courtship index values were measured as the percentage of time spent courting by the subject male, during a $10 \mathrm{~min}$ observation period. The height of each bar indicates the mean $( \pm \mathrm{SE})$ for the corresponding genotype. $B$, The frequency of complete courtship was calculated from the percentage of subject males that attempted copulation with an object fly.

Voila ${ }^{1} / T M 3$ females did not significantly differ for sexual receptivity $(U=708.5 ; n=37 / 44 ; p=0.32)$ or for locomotor activity $(t=0.624 ; \mathrm{df}=82 ; p=0.53)$ when compared with control CS females.

\section{The genetic feminization driven by Voila ${ }^{1}$ yields males with very reduced sexual excitation}

Male flies carrying a single copy of the Voila ${ }^{1}-P G A L 4$ insertion together with the feminizing transgene UAS-transformer (UAStra) (Ferveur et al., 1995) exhibited very little courtship toward females and males: $C I_{\mathrm{f}} / C I_{\mathrm{m}}=3 / 2$ with intact flies and $16 / 15$ with decapitated flies (Fig. 1). Furthermore, Voila ${ }^{1} U A S$-tra males very rarely attempted to copulate with targets of either sex.

The behavioral defect in Voila ${ }^{1} U A S$-tra is not caused by a general alteration of behavior, because the locomotor activities of both Voila ${ }^{1} /$ TM3 and Voila ${ }^{1}$ UAS-tra males are similar $(113 \pm 6$ and $109 \pm 8$ LAU; see Materials and Methods). Moreover, the locomotor activity of both of these male genotypes is significantly higher than for CS males $(68 \pm 4 \mathrm{LAU} ; t=6.45$; df $=93 ; p<$ $0.0001)$.

Voila $^{1} U A S$-tra females were not significantly different from control females $(+/ U A S$-tra; $+/ T M 3)$ either for sexual receptivity $(U=1062 ; n=40 / 58 ; p=0.49)$ or for locomotor activity $(t=$ 0.649 ; df $=56 ; p=0.52$ ).

\section{Mapping the bisexual behavior caused by Voila ${ }^{\mathbf{1}}$}

The PGAL4 insertion in the Voila ${ }^{1}$ strain was mapped in situ, using a GAL4 DNA probe, to the chromosomal region $86 \mathrm{E}_{1-2}$ (Fig. 2A).

Using a set of chromosomal deficiencies $D f(3 R)$ covering the 


\begin{tabular}{|c|c|c|c|c|c|}
\hline \multirow{2}{*}{$\begin{array}{l}\text { Subject fly } \\
\text { (genotype) }\end{array}$} & \multirow{2}{*}{$\begin{array}{l}\text { Object fly } \\
\text { (treatment/sex) }\end{array}$} & \multicolumn{4}{|c|}{ Courtship index (arc sin transformation) } \\
\hline & & Effect & df & $F$ & $p$ \\
\hline \multirow{12}{*}{$\begin{array}{l}\text { Voila/TM3 } \\
\text { (shi) Voila/+ } \\
\text { Canton-S }\end{array}$} & \multirow[t]{3}{*}{ Intact female or male } & Subject & \multirow[t]{3}{*}{205} & 63.71 & 0.00001 \\
\hline & & Object & & 417.80 & 0.00001 \\
\hline & & Interaction & & 1.65 & 0.19 \\
\hline & \multirow{3}{*}{$\begin{array}{l}\text { Decapitated female or } \\
\text { male }\end{array}$} & Subject & \multirow[t]{3}{*}{257} & 74.14 & 0.00001 \\
\hline & & Object & & 223.58 & 0.00001 \\
\hline & & Interaction & & 0.39 & 0.68 \\
\hline & \multirow{3}{*}{$\begin{array}{l}\text { Intact or decapitated } \\
\text { female }\end{array}$} & Subject & \multirow[t]{3}{*}{194} & 55.04 & 0.00001 \\
\hline & & Object & & 23.83 & 0.00002 \\
\hline & & Interaction & & 0.08 & 0.93 \\
\hline & \multirow{3}{*}{$\begin{array}{l}\text { Intact or decapitated } \\
\text { male }\end{array}$} & Subject & \multirow[t]{3}{*}{268} & 78.17 & 0.00001 \\
\hline & & Object & & 1.23 & 0.27 \\
\hline & & Interaction & & 3.59 & 0.03 \\
\hline
\end{tabular}

$\overline{\text { Sex and decapitation of the object were separately tested with a two-factor ANOVA. For genotypes of subject flies, see }}$ Materials and Methods.

region $86 \mathrm{C}$ to $87 \mathrm{C}$ (Fig. $2 \mathrm{~B}$ ), we tested whether the male bisexual orientation observed in the Voila ${ }^{1}$ strain was caused by the PGAL4 insertion. The courtship indices of five males with various $D f(3 R) / T M 3$ genotypes toward control females and males were measured and compared with control w; + /TM3 males (Fig. 2C). The data shown in Table 2 reveal that male flies carrying a single copy of the chromosomal region $86 \mathrm{D}_{4}-\mathrm{E}_{19}$ exhibited abnormal sexual behavior. Among all strains carrying deficiencies, only Kx -1/TM3 and T-32/TM3 males differed significantly from control males in their homo- and heterosexual courtship (Table 2). Furthermore, males from these two strains did not discriminate male and female objects, unlike males carrying other deficiencies that clearly showed a strong heterosexual orientation (Fig. 2C). The significant interaction between both male genotypes $K x-1$ and $T$-32, with regard to the sex of their courted object, suggests that various chromosomal aberrations around the point of Voila insertion can affect male courtship differently. Indeed, we have found that $K x-1$ and $T$-32 males differ for both their hetero- and homosexual courtship (ANOVA; Newman-Keuls post hoc test: $p=0.008$ and 0.029 , respectively).

We also found that Voila homozygotes are lethal, and this defect was also mapped to the chromosome interval $86 \mathrm{D}_{4}-\mathrm{E}_{19}$ (data not shown).

\section{Voila expression pattern in the adult brain and thoracic ganglia}

Using X-gal as a substrate in Voila ${ }^{1} U A S$-lacZ flies, similar patterns of expression were observed in the brain of male and female flies. The most intense staining occurs in Kenyon cells, the intrinsic elements of the MBs (Fig. 3) (Balmer, 1994). All of the Kenyon cell components were clearly visible, including the densely clustered cell bodies in the calyx region and their elaborate fiber pathways in the pedunculus and the MB lobes. Other strongly labeled interneurons were present in the lamina, and a number of other cell bodies and fibers expressed lac $Z$ in the brain and in the subesophageal ganglion (SOG).

Anti- $\beta$-galactosidase immunocytochemistry showed similar patterns and revealed additional elements. Staining was present in a number of cell bodies lateral and ventral to the ALs that form dense arborizations in the AL neuropil. In addition, a few labeled fibers of unknown identity were found in the antennal commis- sure. Outside the ALs, expression was observed in a subset of cells in the pars intercerebralis and their projections in the median bundle, as well as in processes in the giant commissure. Afferent staining in the labial nerve (Fig. 3) (see below) was correlated with label in gustatory centers in the SOG (Stocker and Schorderet, 1981; Nayak and Singh, 1985). The tau reporter gene pattern was similar to the anti- $\beta$-galactosidase pattern and showed in addition that some of the cells ventral to the ALs projected to the inner antennocerebral tract. This suggested expression in relay interneurons that link the ALs with higher brain centers.

In the ventral ganglia, strong labeling of a large number of elements obscured the sensory projections from legs and wings (see below).

\section{Expression pattern in the PNS of the adult head}

In Voila ${ }^{1}$ flies crossed with $U A S$-lacZ, UAS-tau, or $U A S$-GFP, reporter gene expression was observed in specific elements of the head sensory system (Fig. 3). In the labial palps, there was massive label in each taste bristle (Ray et al., 1993) but not in taste pegs (Falk et al., 1976). The simultaneous staining of bristle shafts and afferent axons suggests that reporter gene expression resided in the sensory neurons. However, an additional expression in sheath cells remains possible.

In the pharynx, massive staining was present in the labral sense organ and in the ventral cibarial sense organ (Stocker and Schorderet, 1981; Nayak and Singh, 1983; Balmer, 1994; Stocker, 1994), both of which are putative gustatory sensilla. Less intense label occurred in fishtrap bristles, and there was no expression in the dorsal cibarial sense organ. Staining in certain head nerves was caused by either labeled afferents (e.g., from taste bristles in the labial nerve) or a subset of peripheral nerve glia (e.g., at the base of the maxillary palps or in motor nerve arborizations).

In the third antennal segment, reporters were expressed in all of the trichoid and basiconic sensilla, including their shafts, which were labeled. The tau pattern revealed additional expression in sensilla of the sacculus (Shanbhag et al., 1995) and perhaps in coeloconic sensilla. With none of the reporters was expression visible in afferents from the third segment, suggesting that GAL4 expression is localized in trichogen cells rather than in sensory neurons. In the maxillary palps, expression resided in the basi- 
A

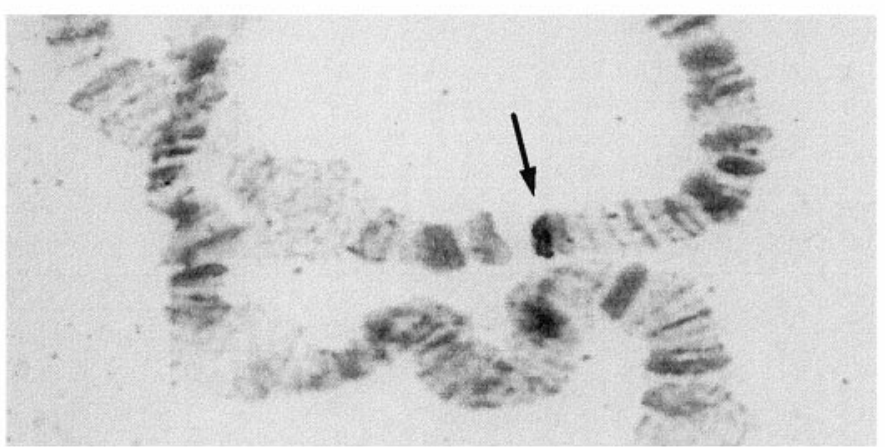

B

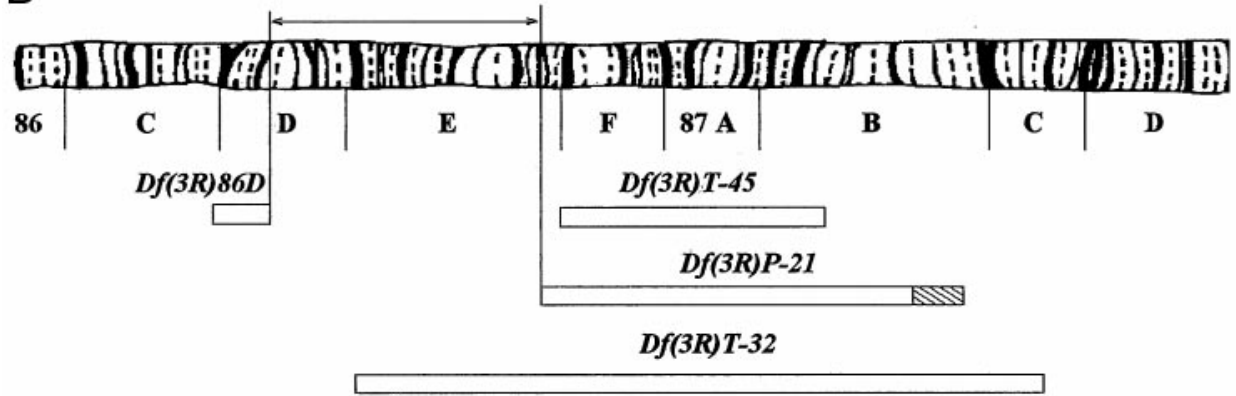

$D f(3 R) K x-1$

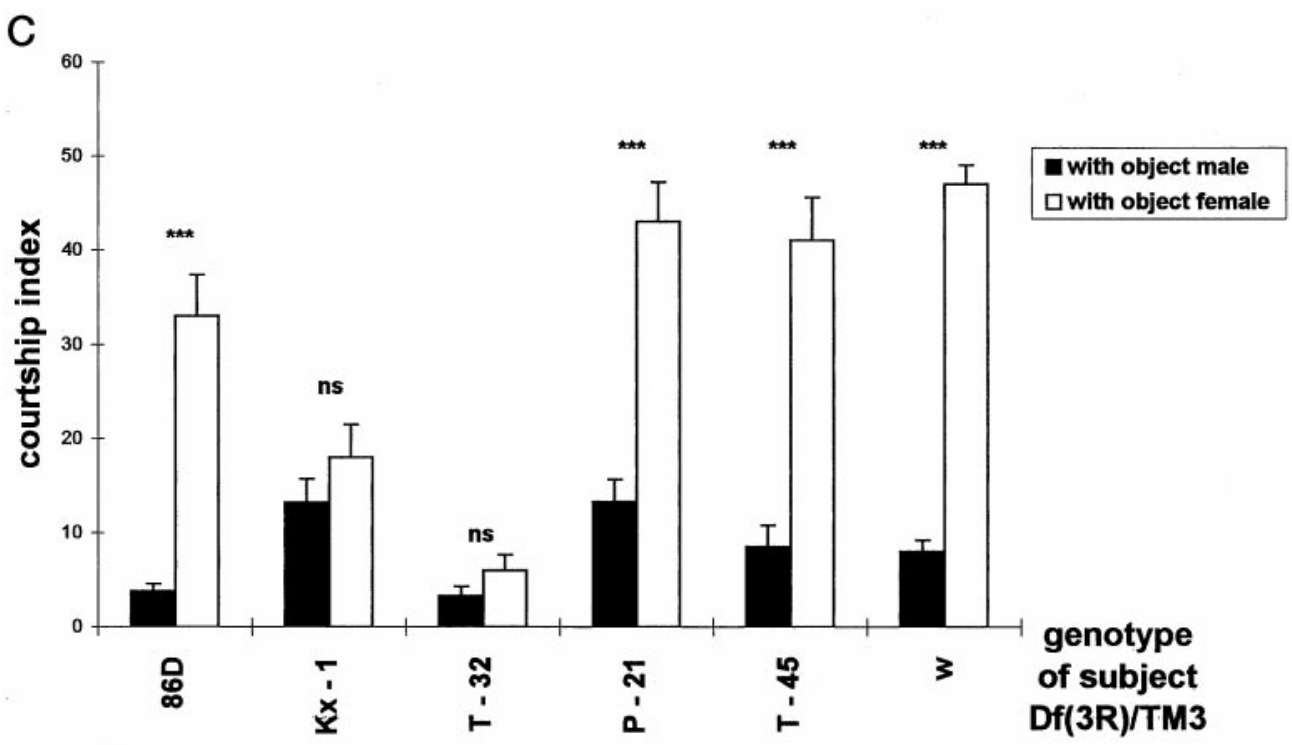

Figure 2. Mapping of Voila ${ }^{1}$. A, In situ chromosomal mapping of the PGAL4 insertion in salivary gland of $w$; Voila $1 /+$ larva. $B$, Deficiencies used to map the bisexual courtship observed in Voila ${ }^{1} / T M 3$ males. Deficiencies are aligned with the salivary gland chromosome map (Lindsley and Zimm, 1992). Bars represent the extent of the deficiencies, and the hatched region represents uncertainty as to breakpoint position. The double-arrowed line indicates the chromosomal region $86 \mathrm{D}_{4}-\mathrm{E}_{19}$. C , Deficiency mapping of the behavioral phenotype of Voila ${ }^{1} / T M 3$. Courtship responses of $w ; D f(3 R) / T M 3$ males toward decapitated male or female targets were measured. The height of each bar indicates the mean $( \pm \mathrm{SE})$ courtship index measured in at least 25 individuals per genotype (15 for $w$; +/TM3). All deficiencies were tested over the TM3, Sb Ser balancer chromosome from the Voila $1 / T M 3$ strain. Sexual discrimination (difference between the intensity of homo- and heterosexual courtship) was statistically tested (two-factor ANOVA, Newman-Keuls test) for each genotype. ${ }^{* * *} p<0.001$. $n s$, Not significant. 


\begin{tabular}{|c|c|c|c|c|c|}
\hline \multirow{2}{*}{$\begin{array}{l}\text { Subject fly } \\
\text { (genotype) }\end{array}$} & \multirow{2}{*}{$\begin{array}{l}\text { Object fly } \\
(\operatorname{sex})\end{array}$} & \multicolumn{4}{|c|}{$\begin{array}{l}\text { Courtship index (arc sin transfor- } \\
\text { mation) }\end{array}$} \\
\hline & & Effect & df & $F$ & $p$ \\
\hline $\begin{array}{l}\text { All } \operatorname{Df}(3) \mathrm{R} \\
\text { deficiencies and } \\
w ;+/ \mathrm{TM} 3\end{array}$ & $\begin{array}{l}\text { Female } \\
\quad \text { or male }\end{array}$ & $\begin{array}{l}\text { Subject } \\
\text { Object } \\
\text { Interaction }\end{array}$ & 349 & $\begin{array}{r}10.97 \\
107.78 \\
7.98\end{array}$ & $\begin{array}{l}0.00001 \\
0.00001 \\
0.00001\end{array}$ \\
\hline $\begin{array}{l}w ; \mathrm{Kx}-1, w ; \mathrm{T}-32 \\
\quad \text { and } w ;+/ \mathrm{TM} 3\end{array}$ & $\begin{array}{l}\text { Female } \\
\quad \text { or male }\end{array}$ & $\begin{array}{l}\text { Subject } \\
\text { Object } \\
\text { Interaction }\end{array}$ & 150 & $\begin{array}{l}18.40 \\
23.72 \\
11.12\end{array}$ & $\begin{array}{l}0.00001 \\
0.00001 \\
0.00003\end{array}$ \\
\hline $\begin{array}{l}w ; 86 \mathrm{D}, w ; \mathrm{P}-21, \\
\quad w ; \mathrm{T}-45, \text { and } w ;+/ \mathrm{TM} 3\end{array}$ & $\begin{array}{l}\text { Female } \\
\quad \text { or male }\end{array}$ & $\begin{array}{l}\text { Subject } \\
\text { Object } \\
\text { Interaction }\end{array}$ & 233 & $\begin{array}{r}2.52 \\
112.31 \\
0.10\end{array}$ & $\begin{array}{l}0.0588 \\
0.00001 \\
0.96\end{array}$ \\
\hline
\end{tabular}

Deficiencies are represented on Figure 2. All object flies were decapitated. Twofactor ANOVA was performed as in Table 1.

conic sensilla (Balmer, 1994) and some of the distal mechanosensory bristles. Again, the lack of stained afferents argues against the presence of GAL4 in sensory neurons. Weak labeling occurred in the Johnston's organ in the second antennal segment.

These data suggest that in olfactory sensilla of the antenna and the maxillary palps, GAL4 is expressed in trichogen or other sheath cells, whereas in the gustatory sensilla of the labial palps and the pharynx, GAL4 expression is neuronal. We found no evidence of sexually dimorphic patterns in these organs.

\section{Expression pattern in wings and legs}

Strong lac $Z$ expression was associated with $\sim 40$ taste bristles on the wing margin (costal vein and radial 1 vein), which are arranged in two rows (Hartenstein and Posakony, 1989; Stocker, 1994). Staining was clearly absent from the mechanosensory bristles, which outnumber the taste bristles by a factor of 4-5 (Fig. 3). Additional labeling occurred in certain wing campaniform sensilla. Label associated with afferents from taste bristles and campaniform sensilla suggests that the expression was neuronal in both cases.

The tibia and tarsus bear two types of bristles, mechanosensory and gustatory (Nayak and Singh, 1983; Nottebohm et al., 1992). On the forelegs, the latter are slightly more abundant in males than in females (Nayak and Singh, 1983). In Voila ${ }^{1} U A S$-lacZ, expression was present exclusively in elements associated with taste bristles (Fig. 4). This correlation was particularly obvious in the tibia, which bears a few well spaced taste bristles among many mechanosensory bristles. Strong staining in the leg nerve suggests that the expression is neuronal rather than associated with sheath cells, similar to gustatory sensilla in the head or wings. The sexual dimorphism of foreleg taste bristles was reflected by a stronger general label of tarsi and of the leg nerve in males.

\section{DISCUSSION}

Of several hundred $P G A L 4$ enhancer-trap strains screened, we focused on the strain carrying the PGAL4 insertion in the Voila locus, which causes two different male courtship behaviors: (1) an increase in bisexual excitation with one copy of the transposon and (2) a loss of heterosexual behavior when PGAL4 drives the feminizing $U A S$-transformer transgene. Bisexual courtship maps to the same location as the PGAL4 insertion $\left(86 \mathrm{E}_{1-2}\right)$.

\section{Is there a relation between bisexual courtship and excitability in Voila ${ }^{1} / T M 3$ males?}

Heterozygous Voila ${ }^{1}$ males generally displayed more vigorous homo- and heterosexual courtship than control strain males.
Their courtship duration with control object females was twice that with control object males. However, their ability to discriminate the sex of their partner was similar to that of control CS males (Table 1). The locomotor activity of Voila ${ }^{1} / T M 3$ males was higher than that of control males, suggesting that their both increased sexual excitation and locomotor activity are caused by their higher excitability. It is possible that a higher locomotor activity simply increases the probability of encounters between sexual partners (Cobb et al., 1987). The fact that Voila ${ }^{1} U A S$-tra males also showed a very high locomotor activity suggests that all males carrying a single copy of Voila ${ }^{1}$ exhibit a hyperactive locomotor activity. However, feminized Voila ${ }^{1} U A S$-tra males had a very reduced courtship, demonstrating that male sexual excitation can be affected without locomotor behavior being altered. These data suggest that both behaviors are related but are controlled by different neural sites.

\section{Mushroom bodies and courtship}

In the CNS, the MBs and to a lesser extent the ALs of Voila ${ }^{1}$ showed GAL4 expression. MBs are known to control courtship and complex behaviors in insects. For example, the ablation of MBs in the male cricket increases its locomotor activity (Huber, 1955). Furthermore, the electric stimulation of a neural structure close to the MBs elicits courtship behavior in male crickets and grasshoppers (Wadepuhl and Huber, 1979). Feminization of MBs in Drosophila can alter pheromone discrimination and male sexual orientation (Ferveur et al., 1995; O’Dell et al., 1995). Our study suggests that the Voila insert increases excitation during male courtship. A difference in the amount of VOILA product (one dose instead of two) - or the accumulation of a mutated product-in the MBs could increase male locomotor activity. We propose that two doses of VOILA are normally required in the MBs to control male-specific behavior. Preliminary results performed both with excisions of the Voila transposon and with deletions covering that locus suggest that the mutating effects of Voila are not related to the dosage of the GAL4 protein (M. Balakireva and J.-F. Ferveur, unpublished data).

\section{Genetic feminization of the peripheral gustatory system in Voila ${ }^{1}$ and reduced heterosexual orientation}

Adult Voila ${ }^{1}$ flies express GAL4 very specifically in the afferent neurons of most gustatory organs: the labial palps, several pharyngeal gustatory organs, and perhaps all the wing and leg taste sensilla. Voila ${ }^{1}$ expression underlines the clear sexual dimorphism in the number of gustatory sensilla on the prothoracic legs (Fig. 4). The gustatory organs on the front legs and the proboscis are thought to play a crucial role during tapping and licking behaviors (Venard et al., 1989; Ferveur and Sureau, 1996; Ferveur, 1997). In contrast, expression of Voila ${ }^{1}$ in olfactory organs does not appear to reside in sensory neurons.

Voila $^{1}$ UAS-tra males show a high locomotor activity but very reduced hetero- and homosexual courtship. Our preliminary data with Voila ${ }^{1} U A S$-tra males show high courtship responses with both male and female targets carrying low levels of or no pheromones. These results suggest that female pheromones, which normally have a stimulatory effect, inhibit the courtship of feminized Voila ${ }^{1}$ $U A S$-tra males. Genetic feminization of the afferent gustatory neurons in Voila ${ }^{1}$ UAS-tra males may have changed their perception properties or may have altered their primary sex-specific projection in the thoracic ganglia (Possidente and Murphey, 1989) or in the brain. It is impossible to measure whether sexual discrimination is altered in the CNS of these males (Ferveur et al., 1995) 

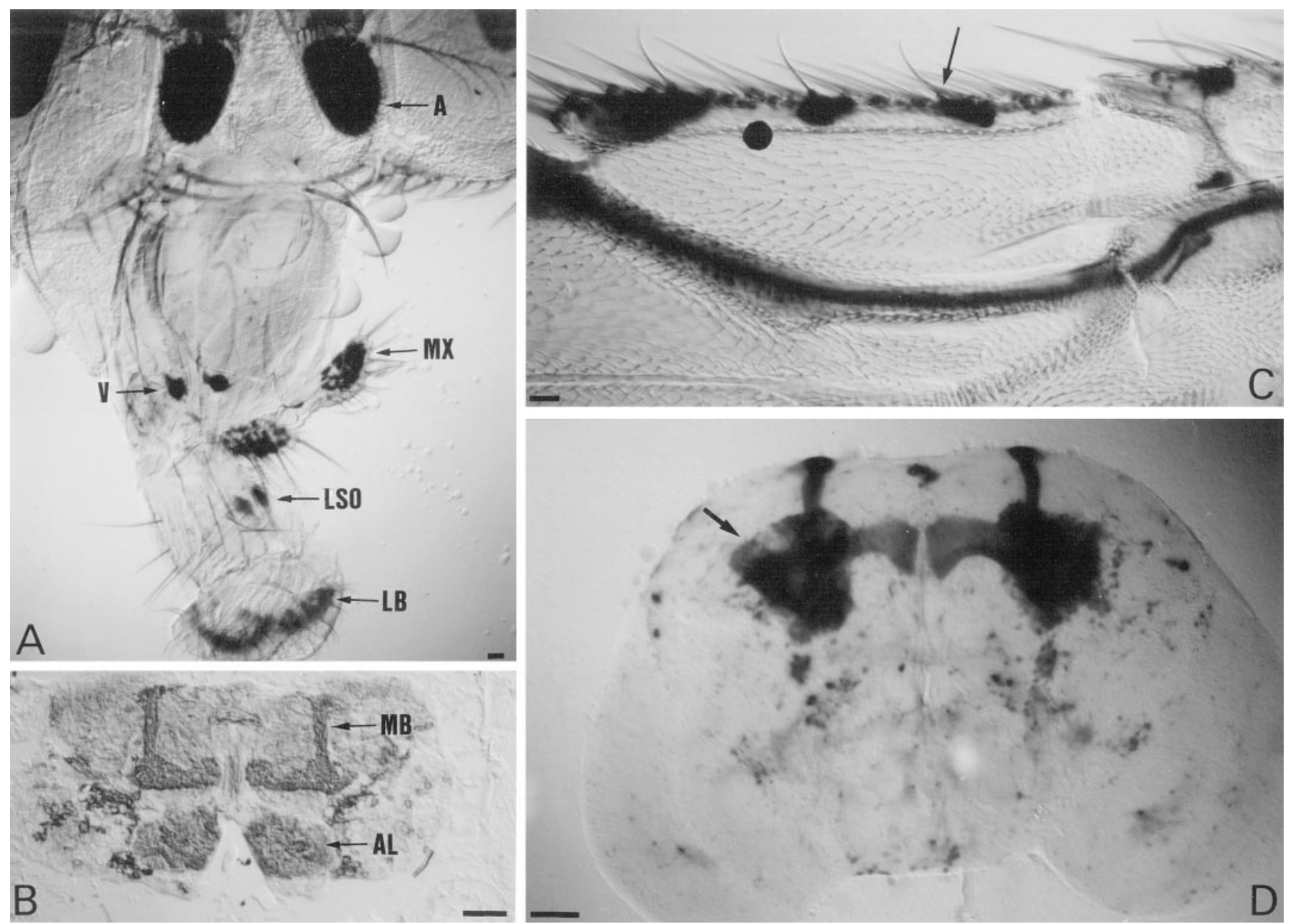

Figure 3. Expression of Voila ${ }^{1}$ in the adult nervous system of 4-d-old flies. $A$, Head cuticle with a complete proboscis. $B$, Frontal section (10 $\mu$ m) of the brain. $C$, Anterior wing margin. $D$, Frontal view of a whole brain. Expression of the $\beta$-galactosidase in Voila ${ }^{1}-U A S$-lacZ males $(A, C, D)$. Anti-tau labeling on Voila ${ }^{1} U A S$-tau male $(B)$. The main peripheral nervous structures (shown on $A$ ) are the third antennal segment $(A)$, the labial palps $(L B)$, the labral sense organ $(L S O)$, the ventral cibarial sense organ $(V)$, and the maxillary palps $(M X)$. The arrow on $C$ indicates neuronal expression associated with a wing gustatory sensillum. In the brain, structures strongly labeled with TAU (shown on $B$ ) are the mushroom bodies (MB; arrow on $D)$ and the antennal lobes $(A L)$. Scale bar, $25 \mu \mathrm{m}$.

because their courtship responses are too weak. However, the influence of the different neural structures in which Voila is expressed can be explored by producing mosaics using the FLP/FRT technique (Golic, 1991).

\section{The behavioral phenotype of Voila is complex}

Male flies carrying various aberrations (deficiency, insertion) in the chromosomal region $86 \mathrm{C}-87 \mathrm{C}$, when compared with control males, showed differences for both their homo- and heterosexual orientation. Further genetic experiments, including PGAL4 remobilization, will help to dissect the influence of the Voila locus on different aspects of male courtship. The comparison of $K x-1$ and $T$-32 deficiencies provides a potential starting point for such a dissection: heterozygous males of either genotype lacking the chromosomal segment $86 \mathrm{E}_{2-19}$ showed very reduced heterosexual excitation and, probably as a consequence, reduced sexual discrimination (Fig. 2). However, the fact that $K x-1$ males showed significantly higher courtship indices than $T-32$ flies suggests that the proximal region $\left(86 \mathrm{D}_{4}-\mathrm{E}_{2}\right)$ of the locus (or its product), which is deleted only in $K x-1$ flies, normally decreases male courtship. We thus predict that heterozygous Voila ${ }^{1} / T M 3$ males, which show high excitation and normal sexual discrimination, are altered only for the proximal part of the Voila locus.

\section{Comparison of Voila $^{\mathbf{1}}$ with other courtship mutations}

In Drosophila melanogaster, some mutant genes have already been shown to alter male courtship behavior. For example, dissatisfaction $(d s f)$ mutant males exhibit a reduced sexual discrimination (Finley et al., 1997). The most intensely studied male courtship mutant is fruitless (fru). Different fru mutant alleles have revealed both genetic and phenotypic complexity at this locus (Villella et al., 1997), as for Voila. The original fru allele ( $\left.f r u^{1}\right)$ shows a decrease in male sexual discrimination (Hall, 1978), whereas a PlacZ targeted allele $\left(\mathrm{fru}^{\text {satori }}\right.$ ) shows almost no sexual excitation or discrimination (Ito et al., 1996). The fru locus codes for several transcripts, some of which are sex-specific and found in restricted neural structures, including the ALs (Ito et al., 1996; Ryner et al., 1996).

Voila $^{1}$ carries a PGAL4 transposon and thus is open to investigation by all the genetic features offered by the PGAL4 enhancertrap system. First, PGAL4 insertion in the Voila ${ }^{1}$ allele has two mutational effects: (1) it is a recessive lethal, and (2) it dominantly enhances hetero- and homosexual courtship behaviors and loco- 

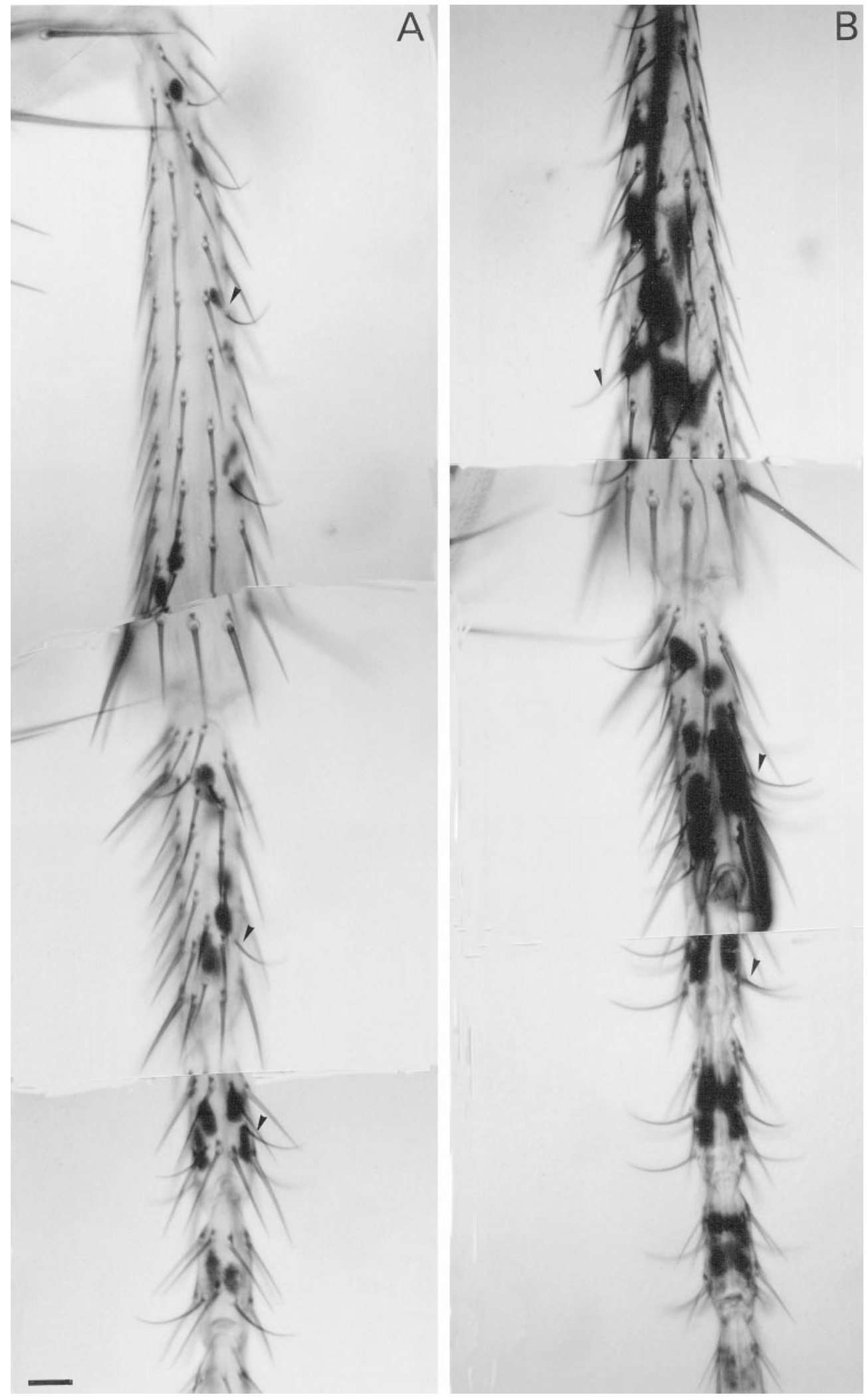

Figure 4. lacZ Expression of Voila ${ }^{1}$ in female $(A)$ and male $(B)$ prothoracic legs. Each picture is a composition showing the tibia (top) and upper tarsal segments (bottom) of Voila ${ }^{1}$ UAS-lacZ 4-dold flies. Arrowheads indicate some of the taste bristles that are always associated with neuronal labeling. Scale bar, $25 \mu \mathrm{m}$. motor activity. Moreover, ectopic expression of the feminizing UAS-tra transgene with Voila ${ }^{1}$ drastically decreases male heterosexual excitation without any other observed behavioral effect. Voila $^{1}$ should be a useful tool for driving the expression of cloned genes to dissect the biological basis of courtship behavior and pheromone perception in Drosophila.

\section{REFERENCES}

Amrein H, Axel R (1997) Genes expressed in neurons of adult male Drosophila. Cell 88:459-469.

Antony C, Jallon J-M (1982) The chemical basis for sex recognition in Drosophila melanogaster. J Insect Physiol 28:873-880.

Balmer D (1994) Isolation von zellspezifisch exprimierenden Gal4- 
Insertionslinien bei Drosophila melanogaster, unter besonderer Berücksichtigung des chemosensorischen Systems. Diploma, University of Fribourg.

Brand AH (1995) GFP in Drosophila. Trends Genet 11:324-325.

Brand AH, Perrimon N (1993) Targeted gene expression as a means of altering cell fates and generating dominant phenotypes. Development 118:401-415.

Cobb MJ, Connolly K, Burnet B (1987) The relationship between locomotor activity and courtship in the melanogaster species sub-group of Drosophila. Anim Behav 35:705-713.

Falk R, Bleiser-Avivi N, Atidia J (1976) Labellar taste organs of Drosophila melanogaster. J Morphol 150:327-342.

Ferveur J-F (1997) The pheromonal role of cuticular hydrocarbons in Drosophila melanogaster. BioEssays 19:353-358.

Ferveur J-F, Sureau G (1996) Simultaneous influence on male courtship of stimulatory and inhibitory pheromones, produced by live sex-mosaic Drosophila melanogaster. Proc R Soc Lond B Biol Sci 263:967-973.

Ferveur J-F, Störtkuhl KF, Stocker RF, Greenspan RJ (1995) Genetic feminization of brain structures and changed sexual orientation in male Drosophila melanogaster. Science 267:902-905.

Ferveur J-F, Savarit F, O'Kane CJ, Sureau G, Greenspan RJ, Jallon J-M (1997) Genetic feminization of pheromones and its behavioral consequences in Drosophila males. Science 276:1555-1558.

Finley KD, Taylor BJ, Milstein M, McKeown M (1997) dissatisfaction, a gene involved in sex-specific behavior and neural development of Drosophila melanogaster. Proc Natl Acad Sci USA 94:913-918.

Golic KG (1991) Site-specific recombination between homologous chromosomes in Drosophila. Science 252:958-961.

Greenspan RJ (1995) Understanding the genetic construction of behavior. Sci Am 272:72-76.

Hall JC (1977) Portions of the central nervous system controlling reproductive behavior in Drosophila melanogaster. Behav Genet 7:291-312.

Hall JC (1978) Courtship among males due to a male-sterile mutation in Drosophila melanogaster. Behav Genet 8:125-141.

Hall JC (1979) Control of male reproductive behavior by the central nervous system of Drosophila: dissection of a courtship pathway by genetic mosaics. Genetics 92:437-457.

Hall JC (1994) The mating of a fly. Science 264:1702-1714.

Hartenstein V, Posakony JW (1989) Development of adult sensilla on the wing and notum of Drosophila melanogaster. Development 107:389-405.

Huber F (1955) Sitz und bedeutung nervöser zentren für instinkthandlungen beim männchen von Gryllus campestris. Z Tierpsychol 12:12-48.

Ito H, Fujitani K, Usui K, Shimizu-Nishikawa K, Tanaka S, Yamamoto D (1996) Sexual orientation in Drosophila is altered by the satori mutation in the sex-determination gene fruitless that encodes a zinc finger protein with a BTB domain. Proc Natl Acad Sci USA 93:9687-9692.

Ito K, Sass H, Urban J, Hofbauer A, Schneuwly S (1997) GAL4responsive $U A S$-tau as a tool for studying anatomy and development of the Drosophila central nervous system. Cell Tissue Res, 290:1-10.

Jallon J-M (1984) A few chemical words exchanged by Drosophila during courtship. Behav Genet 14:441-478.

Lindsley DL, Zimm GG (1992) The genome of Drosophila melanogaster. London: Academic.

Meller VH, Wu KH, Roman G, Kuroda MI, Davis RL (1997) roX1 RNA paints the $\mathrm{X}$ chromosome of male Drosophila and is regulated by the dosage compensation system. Cell 88:445-457.

Nayak SV, Singh RN (1983) Sensilla on the tarsal segments and mouthparts of adult Drosophila melanogaster Meigen (Diptera: Drosophilidae). Int J Insect Morphol Embryol 12:273-291.

Nayak SV, Singh RN (1985) Primary sensory projections from the labella to the brain of Drosophila melanogaster Meigen (Diptera: Drosophilidae). Int J Insect Morphol Embryol 14:115-129.

Nottebohm E, Dambly-Chaudière C, Ghysen A (1992) Connectivity of chemosensory neurons is controlled by the gene poxn in Drosophila. Nature 359:829-832.

O'Dell KMC, Armstrong JD, Yang MY, Kaiser K (1995) Functional dissection of the Drosophila mushroom bodies by selective feminization of genetically defined subcompartments. Neuron 15:55-61.

Possidente DR, Murphey RK (1989) Genetic control of sexually dimor- phic axon morphology in Drosophila sensory neurons. Dev Biol $132: 448-457$

Ray K, Hartenstein V, Rodrigues V (1993) Development of the taste bristles on the labellum of Drosophila. Dev Biol 155:26-37.

Reuter G, Gausz J, Gyurkovics H, Friede B, Bang R, Spierer A, Hall LMC, Spierer P (1987) Modifiers of position-effect variegation in the region from $86 \mathrm{C}$ to $88 \mathrm{~B}$ of the Drosophila melanogaster third chromosome. Mol Gen Genet 210:429-436.

Ryner LC, Goodwin SF, Castrillon DH, Anand A, Villella A, Baker BS, Hall JC, Taylor BJ, Wasserman SA (1996) Control of male sexual behavior and sexual orientation in Drosophila by the fruitless gene. Cell 87:1079-1089.

Shanbhag SR, Singh K, Singh RN (1995) Fine structure and primary sensory projections of sensilla located in the sacculus of the antenna of Drosophila melanogaster. Cell Tissue Res 282:237-249.

Singh RN, Nayak S (1985) Fine structure and primary sensory projections of sensilla on the maxillary palp of Drosophila melanogaster Meigen (Diptera: Drosophilidae). Int J Insect Morphol Embryol 14:291-306.

Smith DP, Shieh B-H, Zuker CS (1990) Isolation and structure of an arrestin gene from Drosophila. Proc Natl Acad Sci USA 87:1003-1007.

Stocker RF (1994) The organization of the chemosensory system in Drosophila melanogaster: a review. Cell Tissue Res 275:3-26.

Stocker RF, Schorderet M (1981) Cobalt filling of sensory projections from internal and external mouthparts in Drosophila. Cell Tissue Res 216:513-523.

Sturtevant AH (1915) Experiments in sexual recognition and the problems of sexual selection in Drosophila. J Anim Behav 5:351-366.

Sturtevant AH (1945) A gene in Drosophila melanogaster that transforms females into males. Genetics 30:297-299.

Taylor BJ, Villella A, Ryner LC, Baker BS, Hall JC (1994) Behavioral and neurobiological implications of sex-determining factors in Drosophila. Dev Genet 15:275-296.

Technau G (1984) Fiber growth and decay in the mushroom bodies of adult Drosophila melanogaster as a function of age and environmental stimulation. J Neurogenet 1:113-126.

Tompkins L, Hall JC (1983) Identification of brain sites controlling female receptivity in mosaics of Drosophila melanogaster. Genetics 103:179-195.

Tompkins L, McRobert SP (1989) Regulation of behavioral aspects of sex determination in Drosophila melanogaster by the Sex-lethal gene. Genetics 123:535-541.

Tompkins L, McRobert SP (1995) Behavioral and pheromonal phenotypes associated with expression of loss-of-function mutations in the Sex-lethal gene of Drosophila melanogaster. J Neurogenet 9:219-226.

Tompkins L, Gross A, Hall JC, Gailey DA, Siegel RW (1982) The role of female movement in the sexual behavior of Drosophila melanogaster. Behav Genet 12:295-307.

Venard R, Antony C, Jallon J-M (1989) Drosophila chemoreceptors. In: Neurobiology of sensory systems (Singh RN, Strausfeld JS, eds), pp 377-385. New York: Plenum.

Villella A, Hall JC (1996) Courtship anomalies caused by doublesex mutations in Drosophila melanogaster. Genetics 143:331-344.

Villella A, Gailey DA, Berwald D, Ohshima S, Barnes PT, Hall JC (1997) Extended reproductive roles of the fruitless gene in Drosophila melanogaster revealed by behavioral analysis of new fru mutants. Genetics 147:1107-1130.

von Schilcher F, Hall JC (1979) Neural topography of courtship song in sex mosaics of Drosophila melanogaster. J Comp Physiol [A] 129:85-95.

Wadepuhl M, Huber F (1979) Elicitation of singing and courtship movements by electrical stimulation of the brain of the grasshopper. Naturwissenschaften 66:320.

Yin Hing AL, Carlson J R (1996) Male-male courtship behavior induced by ectopic expression of the Drosophila white gene: role of sensory function and age. J Neurobiol 30:454-464.

Zhang S-D, Odenwald WF (1995) Misexpression of white $(w)$ gene triggers male-male courtship in Drosophila. Proc Natl Acad Sci USA 92:5525-5529.

Zuker CS, Cowan AF, Rubin GM (1985) Isolation and structure of a rhodopsin gene from Drosophila. Cell 40:851-858. 